ARTIGO ORIGINAL

ORIGINAL ARTICLE

\section{Custo-efetividade e limiar de custo para o uso do sistema intrauterino liberador de levonorgestrel para o tratamento do sangramento uterino anormal sob a perspectiva do Sistema de Saúde Suplementar no Brasil}

\author{
Cost-effectiveness and cost threshold for the use \\ of levonorgestrel-releasing intrauterine system for \\ treatment of heavy menstrual bleeding from the \\ Supplementary Health System perspective in Brazil
}

Natalia Bergamelli Ramos', Johnny Wallef Leite Martins', Ana Tereza de Souza Cruz', Thais Emy Ushikusa', Enzo Asano², Ana Carolina Padula Ribeiro Pereira², Roberta Arinelli Fernandes²

DOI: 10.21115/JBES.v12.n2.p264-72

\section{Palavras-chave:}

menorragia, levonorgestrel, dispositivos intrauterinos, custos e análise de custo, saúde suplementar

\section{RESUMO}

Objetivo: Definir um limiar de custo para o sistema intrauterino liberador de levonorgestrel $52 \mathrm{mg}$ (SIU-LNG 52 mg) para o tratamento do sangramento uterino anormal (SUA) sob a perspectiva do Sistema de Saúde Suplementar brasileiro. Métodos: Foi elaborado um modelo de custo-efetividade para definir o limiar de custo de inserção de SIU-LNG 52 mg em mulheres com SUA em comparação à histerectomia. Um modelo de Markov foi estruturado com ciclos anuais para reproduzir o tratamento do SUA em um e cinco anos, considerando custos médicos diretos e o percentual de histerectomias evitadas como desfecho. O custo da histerectomia foi variado até o valor de 10.000 reais brasileiros (BRL) com incrementos de 500 BRL a cada nova simulação para avaliar o limiar de custo do SIU-LNG 52 mg para igualar o custo total de tratamento de ambas as estratégias analisadas. Resultados: O SIU-LNG 52 mg demonstrou ser uma opção de tratamento dominante quando comparada à histerectomia, levando à redução da frequência de realização do procedimento cirúrgico em 59,62\% das mulheres e redução de 2.557,91 BRL no custo total de tratamento em cinco anos. Ao considerar a histerectomia a 6.000 BRL, o custo do procedimento com SIU-LNG 52 mg poderia ser de até 6.150,35 BRL e 3.994,60 BRL para igualar o custo total de tratamento, em horizontes temporais de um e cinco anos, respectivamente. Conclusão: SIU-LNG 52 mg demonstrou ser uma opção dominante para o manejo do SUA, sendo capaz de atrelar economia para o sistema de saúde a benefícios para a mulher perante a cirurgia.

\section{ABSTRACT}

Objective: To define a cost threshold for the use of levonorgestrel-releasing intrauterine system $52 \mathrm{mg}$ (LNG-IUS 52 mg) for the treatment of heavy menstrual bleeding (HMB) in the Brazilian Supplementary Health System perspective. Methods: A cost-effectiveness model was built to define the cost threshold for insertion of LNG-IUS 52 mg considering women with diagnosis of HMB as the target population and hysterectomy as the comparator. A Markov model was structured with annual cycles

\footnotetext{
Recebido em: 13/11/2020. Aprovado para publicação em: 17/11/2020.

1. Bayer S.A., São Paulo, SP, Brasil.

2. Origin Health Company, São Paulo, SP, Brasil.

Financiamento: Bayer S.A.

Instituição onde o trabalho foi executado: Origin Health Company, São Paulo, SP, Brasil

Declaração de conflitos de interesses: Natália Bergamelli Ramos, Ana Tereza de Souza Cruz, Thais Emy Ushikusa e Johnny Wallef Leite Martins são funcionários da Bayer S.A. Enzo Asano, Roberta Arinelli Fernandes e Ana Carolina Padula Ribeiro Pereira traba-

Iham em uma consultoria que presta serviços à Bayer S.A. e receberam apoio financeiro para participar das análises deste trabalho. Autor correspondente: Natalia Bergamelli Ramos. Rua Alexandre de Gusmão, S/N, Portaria 2, Prédio 401, Bairro Socorro,

São Paulo, SP, Brasil. CEP: 04760-020. Telefone: (11) 5694-4063. E-mail: natalia.ramos@bayer.com
} 
to reproduce HMB treatment in 1 and 5 years, considering direct medical costs and the percentage of avoided hysterectomies as the outcome. Hysterectomy cost was varied up to 10,000 Brazilian real (BRL) with increments of 500 BRL at each new simulation to define LNG-IUS 52 mg cost threshold, to provide equal total treatment costs for both strategies. Results: LNG-IUS $52 \mathrm{mg}$ proved to be a cost-saving option when compared to hysterectomy, leading to a reduction in the frequency of the surgical procedure by $59.62 \%$ and a total treatment cost reduction of 2,557.91 BRL in 5 years. When considering hysterectomy at 6,000 BRL, the cost of the procedure with LNG-IUS $52 \mathrm{mg}$ could be up to 6,150.35 BRL and 3,994.60 BRL to provide equal total treatment cost in 1 and 5 years time horizon, respectively. Conclusion: LNG-IUS 52 mg has proven to be a cost-saving option for the health system in the management of $\mathrm{HMB}$, in addition to the known benefits for women against surgery.

\section{Introdução}

O sangramento uterino anormal (SUA), anteriormente chamado de menorragia, é a denominação utilizada para caracterizar qualquer alteração no ciclo menstrual normal. Essa alteração pode se apresentar por aumento no volume, na duração ou na frequência do ciclo menstrual. A condição pode estar relacionada a causas estruturais, tais como pólipos, adenomiose, leiomioma ou malignidade e hiperplasia, ou ainda a causas não estruturais como coagulopatias, distúrbios da ovulação, disfunção endometrial ou por iatrogenia ou sem causa identificada (Benetti-Pinto et al., 2017; Walker et al., 2020).

O SUA é uma condição observada com alta frequência. Considerando mulheres em diferentes faixas etárias, dentro do período reprodutivo (18-60 anos), a prevalência estimada varia entre 19,9\% e 28,4\% (Fraser et al., 2015; Su et al., 2020; Walker et al., 2020). Já no Brasil, um estudo conduzido na cidade de Pelotas estimou que $46,4 \%$ das mulheres entrevistadas, na faixa etária entre 15 e 54 anos, apresentavam pelo menos um distúrbio menstrual, sendo o sangramento menstrual excessivo o mais prevalente (23,2\%) (Barcelos et al., 2013). Sua ocorrência está relacionada a um impacto importante, reduzindo a qualidade de vida de maneira significativa de acordo com o aumento do volume do sangramento e aumentando a ocorrência de condições como anemia, necessidade de realização de procedimentos cirúrgicos, dor/dismenorreia, absenteísmo, entre outras (Benetti-Pinto et al., 2017; Jensen et al., 2012). Há também um impacto imposto para a sociedade em geral, por meio de uma maior utilização de recursos e custos. Um estudo conduzido nos Estados Unidos estimou que muIheres com SUA apresentam uma diferença de custo de cerca de 2.500 dólares americanos (USD) por ano, quando comparadas a mulheres sem essa condição (Jensen et al., 2012).

O tratamento preconizado para SUA visa reduzir o fluxo menstrual e pode envolver a realização de procedimento cirúrgico ou abordagens medicamentosas. Entre as opções medicamentosas, existem opções não hormonais e hormonais, incluindo a utilização de sistema intrauterino liberador de levonorgestrel 52 mg (SIU-LNG 52 mg) (Benetti-Pinto et al., 2017; Chen et al., 2015). De acordo com as diretrizes da agência britânica National Institute for Health and Care Excellence (NICE), a escolha do tratamento deve considerar as preferências da paciente, a presença de comorbidades, a presença ou ausência de mioma, pólipos, patologia endometrial ou adenomiose e outros sintomas como pressão e dor, e o uso de SIU-LNG 52 mg é recomendado como primeira escolha para mulheres com SUA sem causa identificada, miomas com menos de $3 \mathrm{~cm}$ de diâmetro ou suspeita/diagnóstico de adenomiose. A realização da histerectomia é recomendada apenas em casos de falha, discordância da paciente quanto ao tratamento farmacológico ou na presença de sintomas graves (National Institute of Health and Care Excellence (NICE), 2020). De maneira similar, a Federação Brasileira das Associações de Ginecologia e Obstetrícia (Febrasgo) recomenda a utilização de SIU-LNG 52 mg para SUA por causas não estruturais, sendo o tratamento cirúrgico recomendado apenas após falha com o tratamento clínico (Yela \& BenettiPinto, 2018). No entanto, atualmente, a lista de procedimentos de cobertura obrigatória do Sistema de Saúde Suplementar brasileiro contempla o uso de SIU-LNG apenas como método contraceptivo, sendo, então, a histerectomia o procedimento considerado padrão para tratamento do SUA no sistema privado (Agência Nacional de Saúde Suplementar (ANS), 2018).

A realização de histerectomia está associada ao risco de complicações como infecção, tromboembolismo venoso e sangramento, e, apesar de considerada uma estratégia curativa, promove infertilidade e menopausa precoce (Cheong et al., 2017). Nesse cenário, a utilização de SIU-LNG surge como a opção de tratamento medicamentoso com eficácia na redução do sangramento, além de apresentar benefícios como a manutenção da fertilidade e melhora na qualidade de vida (Davies \& Kadir, 2017).

Diversas publicações apresentam resultados favoráveis quanto ao custo-efetividade do SIU-LNG $52 \mathrm{mg}$ para o manejo do SUA (Blumenthal et al., 2011; Clegg et al., 2007; Da Silva Filho et al., 2016; Escobar-Paredes \& Peralta-Rivera, 2019; Hurskainen et al., 2004; Prota et al., 2020). Dessas, podem-se destacar duas publicações relativas à perspectiva do Sistema de Saúde Suplementar brasileiro que demonstram que o uso de SIU-LNG gera custos diretos e indiretos inferiores e se apresenta como uma alternativa custo-efetiva em comparação ao tratamento cirúrgico, apesar da variabilidade dos valores de reembolso praticados (Da Silva Filho et al., 2016; Prota et al., 2020).

A existência de estudos de custo-efetividade para a perspectiva do Sistema de Saúde Suplementar brasileiro pode auxiliar na tomada de decisão em realidades com custos 
semelhantes aos adotados nos estudos. Porém, é importante ressaltar que esses resultados podem não ser passíveis de extrapolação para todas as operadoras de saúde, uma vez que cada operadora pode apresentar realidades de custo diferentes.

Visando permitir essa extrapolação dos custos e resultados para diferentes realidades de cada operadora, o limiar de custo surge como uma estratégia matemática que estima o custo máximo com a nova estratégia de tratamento para que haja equivalência econômica entre as opções terapêuticas avaliadas. Assim, o objetivo deste artigo é analisar o limiar de custo para o procedimento com SIU-LNG no tratamento do SUA em comparação à histerectomia, no contexto do Sistema de Saúde Suplementar brasileiro.

\section{Métodos}

No presente estudo, uma análise de custo-efetividade foi desenvolvida com o objetivo de definir o limiar de custo de inserção de SIU-LNG 52 mg em mulheres com SUA, em comparação à histerectomia. Para isso, foram analisados os custos do dispositivo e de seu procedimento de inserção visando obter equivalência econômica entre as estratégias de tratamento, isto é, custo total de tratamento incremental igual a zero.

O único tratamento atualmente disponível para manejo do SUA, no Sistema de Saúde Suplementar, é a histerectomia, portanto essa foi a estratégia considerada como comparador, incluindo os custos médicos diretos, sob essa perspectiva (Agência Nacional de Saúde Suplementar (ANS), 2018). A análise contou com horizontes temporais de um e cinco anos a partir da idade inicial média de 43 anos, conforme descrito em Heliövaara-Peippo et al. (2013) (HeliövaaraPeippo et al., 2013).

\section{Estrutura do modelo de custo-efetividade}

A estrutura da análise de custo-efetividade foi desenvolvida por meio de um modelo analítico de decisão de Markov com ciclos anuais para reproduzir o tratamento do SUA ao longo de um horizonte de tempo de até cinco anos. O desfecho de saúde utilizado foi o percentual de "histerectomias evitadas", ou seja, o percentual de cirurgias evitadas ao longo do tratamento do SUA utilizando SIU-LNG 52 mg. Os desfechos econômicos considerados foram os custos médicos diretos, incluindo os recursos médicos utilizados diretamente para o tratamento e acompanhamento da paciente, como exames, diárias hospitalares, materiais e procedimentos (Associação Médica Brasileira (AMB), 2018; Escobar-Paredes \& Peralta-Rivera, 2019; Ministério da Saúde (Brasil), 2020; Secretaria da Administração do Estado da Bahia, 2018).

A taxa de desconto de 5\% ao ano foi aplicada para custos e desfechos. Os custos indiretos, como aqueles relacionados à perda de produtividade da paciente associada à patologia, não foram contemplados na análise (Ministério da Saúde (Brasil), 2014).
Para a estratégia de tratamento com SIU-LNG 52 mg, o modelo é composto de quatro estados de saúde mutuamente excludentes (Figura 1). As pacientes iniciam o tratamento no estado "em tratamento com SIU-LNG 52 mg"; enquanto permanecem nesse estado, continuam em uso de SIU-LNG 52 mg. A qualquer momento, as pacientes podem evoluir ou não para os demais estados de saúde de maneira unidirecional, isto é, não é permitido o retorno ao estado de saúde prévio. Quando não há controle do sangramento com SIU-LNG 52 mg, as pacientes interrompem o tratamento com o dispositivo e realizam a histerectomia, migrando para o estado "histerectomia", no qual permanecem até o final do horizonte temporal ou até a morte. No estado de saúde "sem tratamento ativo", a paciente interrompe o tratamento com SIU-LNG 52 mg, sendo essa interrupção causada pela descontinuação sem a necessidade de realizar uma histerectomia ou pela ocorrência da menopausa, que consequentemente interrompe a necessidade do tratamento do SUA. Ainda no estado "sem tratamento ativo", a paciente está sujeita a realizar a histerectomia em caso de recorrência do SUA e permanecer nesse estado até o final do horizonte temporal, ou até a ocorrência da morte.

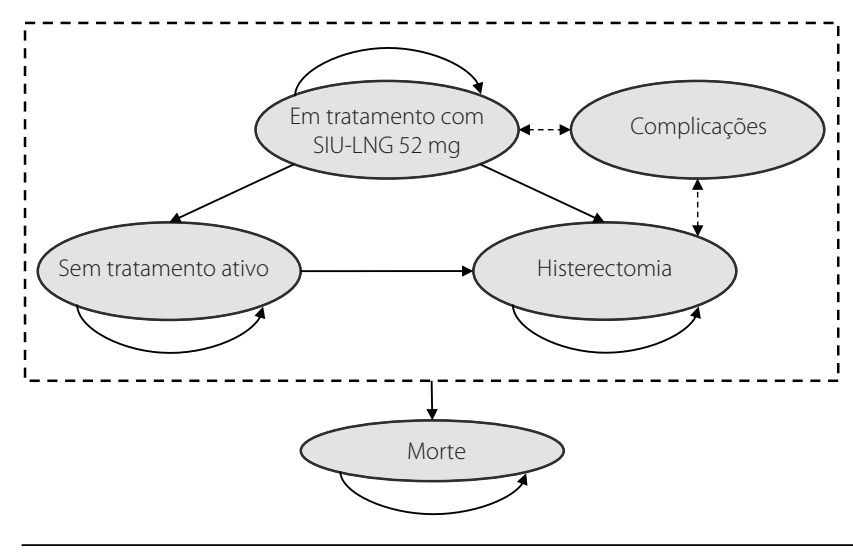

Figura 1. Desenho do modelo.

Para a estratégia de tratamento com histerectomia, o modelo é composto de dois estados de saúde mutuamente excludentes: "histerectomia" e "morte". Após realizar a histerectomia, a paciente é acompanhada até o fim do horizonte temporal ou morte.

Para pacientes em tratamento com SIU-LNG 52 mg, foram incluídas as complicações de expulsão espontânea do dispositivo e perfuração do útero, parcial ou total, considerando que elas ocorrem no primeiro ano após a inserção ou reinserção do dispositivo (Barnett et al., 2017; Heinemann et al., 2015).

Após a histerectomia, foram consideradas as complicações relacionadas à cirurgia, sendo elas computadas uma vez no ciclo em que a histerectomia ocorre (Hurskainen et al., 2004). 


\section{Dados de eficácia}

Os parâmetros de eficácia utilizados na análise de custo-efetividade foram obtidos do estudo de Heliövaara-Peippo et al. (2013). As probabilidades anuais de descontinuação do SIU-LNG 52 mg e de ocorrência de histerectomia utilizadas no cálculo estão demonstradas na Tabela 1 (Heliövaara-Peippo et al., 2013). As ocorrências de complicações relacionadas à histerectomia foram obtidas do estudo de Hurskainen et al. (2004), estudo antecessor do Heliövaara-Peippo et al. (2013) (Heliövaara-Peippo et al., 2013; Hurskainen et al., 2004).

O estudo de Heliövaara-Peippo et al. (2013) é um ensaio clínico randomizado que avaliou o tratamento do SUA com SIU-LNG 52 mg e histerectomia ao longo de 10 anos de tratamento. Nesse estudo, 117 pacientes iniciaram o tratamento com SIU-LNG 52 mg. Dessas, 81, 57 e 44 permaneceram em tratamento em 1, 5 e 10 anos, respectivamente. Ainda, 24, 50 e 55 pacientes realizaram a histerectomia em 1,5 e 10 anos, respectivamente. É importante ressaltar que os dados utilizados apresentam sobreposição entre o número de pacientes que descontinuaram o tratamento com SIU-LNG 52 mg e o número de pacientes que realizaram histerectomia. Após um ano de tratamento, 36 pacientes descontinuaram o tratamento com SIU-LNG 52 mg, e 24 dessas foram submetidas a histerectomia. Por outro lado, 12 pacientes que descontinuaram o tratamento não realizaram histerectomia (Heliövaara-Peippo et al., 2013). O estudo de Heinemann et al. (2015) avaliou o risco de perfuração uterina com o uso de SIU-LNG 52 mg, apontando a ocorrência desse evento em 1,4 a cada mil pacientes $(0,14 \%)$, com $80 \%$ de perfurações totais e $20 \%$ de parciais. Já no estudo de Barnett et al. (2018), a expulsão espontânea do SIU-LNG 52 mg ocorreu em 1,4\% das mulheres (Barnett et al., 2017; Heinemann et al., 2015).

Para ambos os braços comparados - SIU-LNG 52 mg e histerectomia -, a probabilidade de morte foi obtida por meio da tábua de mortalidade publicada pelo Instituto Brasileiro de Geografia e Estatística (IBGE), apresentando dados de mortalidade da população geral segmentada por sexo (Instituto Brasileiro de Geografia e Estatística (IBGE), 2018).

\section{Uso de recursos}

Os recursos de saúde utilizados na análise de custo-efetividade referem-se aos custos associados ao uso do SIU-LNG $52 \mathrm{mg}$, cirurgia de histerectomia, acompanhamento das pacientes e manejo de complicações. Os padrões de utilização de recursos para acompanhamento e manejo das pacientes foram estimados de acordo com a opinião de especialistas e custeados por meio da Classificação Brasileira Hierarquizada de Procedimentos Médicos (CBHPM) versão 2018, com o valor de porte atualizado de 2019, do Sistema de Assistência à Saúde dos Servidores Públicos Estaduais (Planserv) e da Lista de Preços da Câmara de Regulação do Mercado de Medicamentos (CMED) (Associação Médica Brasileira (AMB), 2018; Ministério da Saúde (Brasil), 2020; Secretaria da Administração do Estado da Bahia, 2018).

Os custos considerados incluem consultas, exames, procedimentos, materiais, e diárias necessárias para o tratamento e acompanhamento da paciente.

Para o cenário-base, o custo de utilização considerado para SIU-LNG 52 mg foi de 1.468,98 reais brasileiros (BRL) por inserção, composto pelo Preço Fábrica com 18\% de ICMS (PF 18\%) do dispositivo (887,46 BRL) e pelo procedimento de inserção, de acordo com CBHPM (581,52 BRL) (Associação Médica Brasileira (AMB), 2018; Ministério da Saúde (Brasil), 2020). Para a retirada do SIU-LNG 52 mg, dada a indisponibilidade de um procedimento específico, foi considerado o valor de uma consulta médica (167,43 BRL) (Associação Médica Brasileira (AMB), 2018). Adicionalmente, para o cenário-base do modelo, adotou-se o custo de histerectomia apresentado por Prota et al. (2020), que avaliou o custo-efetividade de SIU-LNG 52 mg em comparação com a histerectomia, sob a perspectiva da Unimed Campinas, de 1.417,84 USD (Prota et al., 2020). A conversão considerou a cotação média do dólar referente ao período de 1 a 31 de dezembro de 2019 (1 $\mathrm{USD}=4,1089 \mathrm{BRL})$, já que esse se refere ao período aproximado de submissão do estudo, cuja data-limite para envio foi em janeiro de 2020. Ainda, após a conversão, ajustou-se o valor pelo Índice de Preço ao Consumidor para Saúde (IPCSaúde) acumulado entre dezembro de 2019 e agosto de 2020 (2,80\%), resultando em um custo de histerectomia de

Tabela 1. Probabilidades anuais de descontinuação e histerectomia

\begin{tabular}{|c|c|c|c|c|}
\hline Período & Em tratamento & Descontinuação (\%) & Histerectomias & $\begin{array}{c}\text { Ocorrência de } \\
\text { histerectomia (\%) }\end{array}$ \\
\hline Baseline & $117^{*}$ & & $0^{*}$ & \\
\hline 1 ano & $81 *$ & 30,77 & $24^{*}$ & 20,51 \\
\hline 2 anos & 74 & 8,41 & 31 & 9,22 \\
\hline 3 anos & 68 & 8,41 & 38 & 9,22 \\
\hline 4 anos & 62 & 8,41 & 45 & 9,22 \\
\hline 5 anos & $57^{*}$ & 8,41 & $50 *$ & 9,22 \\
\hline
\end{tabular}

* Valores extraídos do estudo Heliövaara-Peippo et al. (2013) (Heliövaara-Peippo et al., 2013). 
5.988,92 BRL (Fundação Instituto de Pesquisas Econômicas (Fipe), 2020; Ministério da Fazenda (Brasil), 2020).

Além disso, os custos referentes às complicações associadas à histerectomia e ao uso do SIU-LNG $52 \mathrm{mg}$ foram incluídos na análise por meio da metodologia de microcusteio. As complicações consideradas associadas à histerectomia foram: dor abdominal, febre, fístula vesicovaginal, íleo paralítico, infecção pélvica, oclusão intestinal, peritonite, retenção urinária, ruptura da ferida cirúrgica, infecção da ferida, sangramento perioperatório, sangramento pós-operatório e lesão no ureter (Hurskainen et al., 2004). Para o uso do SIU-LNG 52 mg, as complicações consideradas foram a expulsão espontânea do dispositivo e a perfuração uterina (Barnett et al., 2017; Heinemann et al., 2015).

Para a análise de limiar de custo, o custo da cirurgia de histerectomia foi variado de 1.000,00 BRL até o valor de 10.000,00 BRL com incrementos de 500,00 BRL a cada nova simulação para avaliar o limiar de custo do SIU-LNG. Assim, chegou-se ao valor limiar de SIU-LNG 52 mg necessário para igualar o custo de tratamento de ambas as estratégias analisadas em função do custo da cirurgia de histerectomia e do horizonte temporal analisado.

\section{Resultados}

\section{Análise de custo-efetividade}

A Tabela 2 apresenta os valores de cada parâmetro de custo em cada intervenção. O tratamento com SIU-LNG 52 mg envolve custo de dispositivo de 887,46 BRL, custo de inserção do dispositivo de 581,52 BRL, custo de remoção do dispositivo de 167,43 BRL e custo de acompanhamento anual de 430,91 BRL. Por outro lado, a histerectomia está associada a custo de cirurgia de $5.988,92 \mathrm{BRL}$, custo de primeiro ano de acompanhamento de 959,90 BRL e custo de acompanhamento de anos subsequentes de 167,43 BRL.

Conforme descrito anteriormente, a histerectomia está associada a 13 diferentes complicações, gerando um custo total de complicações de 1.296,77 BRL após ponderação dos respectivos custos pelas frequências de ocorrência de cada complicação. Já o tratamento com SIU-LNG 52 mg está associado às complicações de perfuração uterina e expulsão espontânea do dispositivo, acarretando um custo total de complicação de 24,31 BRL após ponderação pelas frequências (Tabela 3).

A Tabela 4 mostra o resultado da análise de custo-efetividade para os horizontes de um e cinco anos, demonstrando que o SIU-LNG 52 mg se mantém dominante em relação à histerectomia em ambos os horizontes temporais avaliados. Isso acontece, uma vez que o SIU-LNG 52 mg apresenta um custo total inferior ao custo total da histerectomia e uma porcentagem de histerectomia inferior.
Tabela 2. Custo de histerectomia e SIU-LNG 52 mg

\begin{tabular}{lc}
\hline Itens de custo & Custo (BRL) \\
\hline SIU-LNG $\mathbf{5 2} \mathbf{~ m g}$ & \\
\hline Dispositivo & $887,46^{*}$ \\
\hline Inserção do dispositivo & $581,52 \dagger$ \\
\hline Remoção do dispositivo & $167,43 \dagger$ \\
\hline Acompanhamento anual & $430,91 \dagger$ \\
\hline Histerectomia & \\
\hline Cirurgia & $5.988,92^{\ddagger}$ \\
\hline Primeiro ano de acompanhamento & $959,90^{\dagger}$ \\
\hline Anos subsequentes de acompanhamento & $167,43^{\dagger}$ \\
\hline
\end{tabular}

* Lista de Preços CMED considerando o Preço Fábrica com a alíquota de 18\% de ICMS (Ministério da Saúde (Brasil), 2020).

+ Microcusteio com custos da CBHPM 2018 com porte atualizado para 2019 (Associação Médica Brasileira (AMB), 2018).

\# Prota et al., 2019 (Prota et al., 2020).

Tabela 3. Complicações relacionadas a histerectomia e SIU-LNG $52 \mathrm{mg}$

\begin{tabular}{|c|c|c|c|}
\hline Complicações & $\begin{array}{l}\text { Histerectomia } \\
\text { (\%) }\end{array}$ & $\begin{array}{l}\text { SIU-LNG } \\
52 \mathrm{mg}(\%)\end{array}$ & $\begin{array}{c}\text { Custo de } \\
\text { evento (BRL) }\end{array}$ \\
\hline Infecção pélvica & $9,43^{*}$ & & $7.013,87^{\S}$ \\
\hline Dor abdominal & $3,77^{*}$ & & $598,34^{\S}$ \\
\hline Infecção da ferida & $8,81^{*}$ & & $1.357,33^{\S}$ \\
\hline $\begin{array}{l}\text { Sangramento } \\
\text { perioperatório }\end{array}$ & $0,63^{*}$ & & $10.955,78^{\S}$ \\
\hline Oclusão intestinal & $0,63^{*}$ & & $7.094,41^{\S}$ \\
\hline $\begin{array}{l}\text { Sangramento } \\
\text { pós-operatório }\end{array}$ & $1,89^{*}$ & & $6.906,09^{\S}$ \\
\hline Febre & $1,89^{*}$ & & $2.927,89^{\S}$ \\
\hline Retenção urinária & $3,14^{*}$ & & $673,96^{5}$ \\
\hline Íleo paralítico & $1,26^{*}$ & & $1.235,61^{\S}$ \\
\hline Ruptura da ferida & $1,26^{*}$ & & $402,03^{\S}$ \\
\hline Peritonite & $0,63^{*}$ & & $7.359,46^{\S}$ \\
\hline Lesão no ureter & $0,63^{*}$ & & $10.626,56^{\S}$ \\
\hline Fístula vesicovaginal & $0,63^{*}$ & & $6.202,50^{\S}$ \\
\hline Perfuração uterina & & $0,14^{+}$ & $2.673,37^{\S}$ \\
\hline Expulsão espontânea & & $1,40^{\ddagger}$ & $1.468,98^{\|}$ \\
\hline
\end{tabular}

* Hurskainen et al. (2004) (Hurskainen et al., 2004).

${ }^{+}$Heineman et al. (2015) (Heinemann et al., 2017).

* Barnett et al. (2018) (Barnett et al., 2017).

$\S$ Microcusteio com custos da CBHPM 2018 com porte atualizado para 2019, Planserv, Lista de Preços CMED considerando o Preço Fábrica com a alíquota de 18\% de ICMS (Associação Médica Brasileira (AMB), 2018; Ministério da Saúde (Brasil), 2020; Ministério da Saúde (Brasil), 2020; Secretaria da Administração do Estado da Bahia, 2018; Secretaria da Administração do Estado da Bahia, 2018).

"Considerado o custo da reinserção do SIU-LNG. 
Tabela 4. Resultados de custo e efetividade incremental por horizonte de tempo

\begin{tabular}{lcc}
\hline \multirow{2}{*}{ Desfechos } & \multicolumn{2}{c}{$\begin{array}{c}\text { Resultados incrementais } \\
\text { (SIU-LNG 52 } \mathbf{~ m g ~ v e r s u s ~ H i s t e r e c t o m i a ) ~}\end{array}$} \\
\cline { 2 - 3 } Custos & $\mathbf{1}$ ano & $\mathbf{5}$ anos \\
\hline SIU-LNG 52 mg & $-R \$ 4.744,65$ & $-R \$ 2.557,91$ \\
\hline Histerectomia & $\mathrm{R} \$ 1.543,02$ & $\mathrm{R} \$ 1.573,40$ \\
\hline Acompanhamento & $-\mathrm{R} \$ 4.762,82$ & $-\mathrm{R} \$ 3.570,81$ \\
\hline Complicações & $-\mathrm{R} \$ 497,31$ & $\mathrm{R} \$ 208,93$ \\
\hline $\begin{array}{l}\text { \% Histerectomias } \\
\text { evitadas }\end{array}$ & $-\mathrm{R} \$ 1.027,54$ & $-\mathrm{R} \$ 769,44$ \\
\hline $\begin{array}{l}\text { RCEl por } \\
\text { histerectomia evitada }\end{array}$ & $-79,53 \%$ & $-59,62 \%$ \\
\hline
\end{tabular}

\section{Análise de limiar de custo}

A Tabela 5 apresenta o limiar de custo de SIU-LNG 52 mg de acordo com a variação do custo de histerectomia para os horizontes temporais de um e cinco anos. Ao considerar a histerectomia a 6.000 BRL, valor próximo ao do cenário-base do modelo de custo-efetividade (Prota et al., 2020), obtêm-se aos limiares de SIU-LNG 52 mg de 6.150,35 BRL e 3.994,60 BRL, para os horizontes temporais de um e cinco anos, respectivamente. Isto é, o custo do dispositivo mais procedimento de inserção de SIU-LNG 52 mg poderia somar 3.994,60 BRL para que o custo total de tratamento do SUA ao longo de cinco anos com esse dispositivo tivesse o mesmo custo total que a adoção da histerectomia para esse tratamento.

É possível notar que o aumento do horizonte temporal resultou em uma diminuição do limiar de custo do SIU-LNG (Tabela 5), o que reflete a influência do custo de acompanhamento anual e realização de histerectomias associados ao uso do dispositivo, em comparação à diluição do custo inicial da cirurgia em pacientes tratadas com histerectomia.

A Figura 2 apresenta o limiar de custo de SIU-LNG 52 mg em função do custo de histerectomia. O eixo horizontal representa o custo da cirurgia, enquanto o eixo vertical representa o valor máximo que o SIU-LNG 52 mg mais procedimento de inserção podem totalizar para que essa ainda seja uma opção de tratamento dominante em relação à histerectomia, o que significa que qualquer valor abaixo desse limiar resultaria em uma economia no custo total de tratamento do SUA em comparação à histerectomia.

Por exemplo, para o horizonte temporal de um ano, considerando um custo de histerectomia de $6.000 \mathrm{BRL}$, o custo do dispositivo SIU-LNG 52 mg mais procedimento de inserção poderia totalizar até $6.150,35$ BRL para que essa alternativa de tratamento ainda seja dominante na análise de custo-efetividade.
Tabela 5. Limiar de custo de SIU-LNG 52 mg, considerando dispositivo e inserção, versus custo de histerectomia para que ambas as estratégias de tratamento tenham o mesmo custo total de tratamento do SUA ao longo de até 5 anos

\begin{tabular}{|c|c|c|}
\hline \multirow{2}{*}{$\begin{array}{l}\text { Custo de histerectomia } \\
\text { (BRL) }\end{array}$} & \multicolumn{2}{|c|}{$\begin{array}{l}\text { Limiar de custo de SIU-LNG } 52 \text { mg por } \\
\text { horizonte de tempo (BRL) }\end{array}$} \\
\hline & 1 ano & 5 anos \\
\hline $1.000,00$ & $2.234,30$ & $1.058,64$ \\
\hline $1.500,00$ & $2.625,91$ & $1.352,24$ \\
\hline $2.000,00$ & $3.017,51$ & $1.645,83$ \\
\hline $2.500,00$ & $3.409,12$ & $1.939,43$ \\
\hline $3.000,00$ & $3.800,72$ & $2.233,03$ \\
\hline $3.500,00$ & $4.192,33$ & $2.526,62$ \\
\hline $4.000,00$ & $4.583,93$ & $2.820,22$ \\
\hline $4.500,00$ & $4.975,54$ & $3.113,81$ \\
\hline $5.000,00$ & $5.367,14$ & $3.407,41$ \\
\hline $5.500,00$ & $5.758,75$ & $3.701,01$ \\
\hline $6.000,00$ & $6.150,35$ & $3.994,60$ \\
\hline $6.500,00$ & $6.541,96$ & $4.288,20$ \\
\hline $7.000,00$ & $6.933,56$ & $4.581,80$ \\
\hline $7.500,00$ & $7.325,17$ & $4.875,39$ \\
\hline $8.000,00$ & $7.716,77$ & $5.168,99$ \\
\hline $8.500,00$ & $8.108,38$ & $5.462,59$ \\
\hline $9.000,00$ & $8.499,98$ & $5.756,18$ \\
\hline $9.500,00$ & $8.891,59$ & $6.049,78$ \\
\hline $10.000,00$ & $9.283,19$ & $6.343,38$ \\
\hline
\end{tabular}

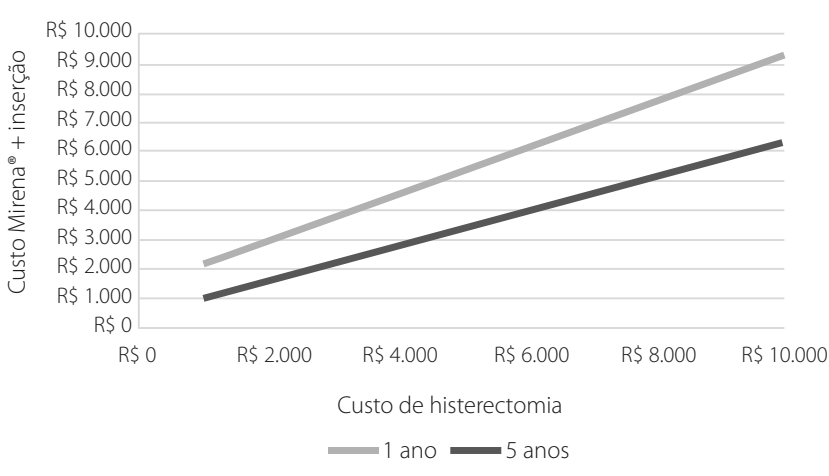

Figura 2. Limiar de custo: SIU-LNG 52 mg versus histerectomia.

\section{Discussão}

Este estudo foi conduzido com o objetivo de definir um limiar de custo para a utilização de SIU-LNG 52 mg como opção terapêutica para manejo do SUA no contexto do Sistema de Saúde Suplementar brasileiro. Considerando que existe uma variabilidade no custo de recursos em saúde entre as operadoras que compõem o Sistema de Saúde Suplementar brasileiro que impossibilita a extrapolação de achados de estudos 
disponíveis na literatura, os resultados de limiares de custo ora apresentados são de fundamental importância para auxiliar no processo de tomada de decisão no cenário nacional.

Estudos que visam entender a relação entre benefícios clínicos e custos associados à adoção de diferentes tecnologias são essenciais para auxiliar no processo decisório em relação às alternativas de tratamento disponíveis (Secoli et al., 2010). Nesse cenário, esta análise propõe estimar limiares de custo nos quais o SIU-LNG 52 mg se mantenha dominante em relação ao comparador utilizado: a histerectomia.

A estimativa de um limiar de custo apresenta como vantagem a extrapolação dos resultados para diferentes realidades locais desejadas, uma vez que a análise contempla a variação do custo de tratamento objeto de comparação. Outros autores utilizaram essa abordagem para avaliar a utilização de tecnologias (Culhane et al., 2020; Maulsby et al., 2019). No entanto, não foram localizados trabalhos visando determinar um limiar de custo para SIU-LNG 52 mg no manejo do SUA para fins de comparação dos resultados.

Os limiares de custo de SIU-LNG 52 mg foram de 6.150,35 BRL e 3.994,60 BRL, para os horizontes temporais de um e cinco anos, respectivamente, em comparação à histerectomia, considerando-se um custo de 6.000,00 BRL por cirurgia realizada. Em suma, caso o custo de SIU-LNG 52 mg, considerando o dispositivo e a inserção, totalizasse um valor menor do que esses limiares, a implementação do SIU-LNG 52 mg como estratégia de tratamento resultaria em uma economia no custo total de tratamento do SUA em comparação à histerectomia.

A análise de custo-efetividade apresentada neste estudo em um horizonte temporal de até cinco anos demonstrou que o uso de SIU-LNG $52 \mathrm{mg}$ gera economia de recursos, além de ganhos em termos de eficácia, quando comparado à realização de histerectomia para o manejo do SUA no contexto da saúde suplementar. Essa conclusão é determinada uma vez que o custo total de tratamento é inferior àquele observado com a realização de histerectomia $(6.278,28$ BRL versus $8.836,19$ BRL), além de uma redução de $59,62 \%$ dos procedimentos cirúrgicos. Um menor custo em pacientes que utilizaram SIU-LNG 52 mg, quando comparado àquele observado em pacientes submetidos à histerectomia, foi previamente relatado no estudo de Heliövaara-Peippo et al. (2013), conduzido sob a perspectiva do sistema de saúde finlandês (Heliövaara-Peippo et al., 2013).

Outras análises foram conduzidas, também com a intenção de avaliar o custo-efetividade de SIU-LNG 52 mg para manejo do SUA, e demonstraram que essa é uma opção custo-efetiva, quando comparada ao tratamento cirúrgico (Blumenthal et al., 2011; Louie et al., 2017; Spencer et al., 2017). Considerando o cenário nacional, alguns estudos demonstram que SIU-LNG 52 mg e a realização de histerectomia são eficazes no manejo do SUA e que o dispositivo uterino demanda uma menor utilização de recursos e custos
(Bahamondes et al., 2012; Da Silva Filho et al., 2016; Prota et al., 2020; Teich et al., 2012). Ao analisar especificamente o custo-efetividade de SIU-LNG 52 mg para manejo do SUA na perspectiva da saúde suplementar, os resultados corroboram os achados apresentados nesta análise, sendo a tecnologia capaz de gerar uma economia de até 1,8 milhão de dólares nos primeiros cinco anos de utilização (Prota et al., 2020).

Além dos custos diretos relacionados à presença do SUA, o diagnóstico da condição está associado a um importante custo indireto, com diferenças significativas para presenteísmo e absenteísmo em relação a mulheres sem a condição (Hasselrot et al., 2018). A utilização de SIU-LNG 52 mg demonstrou previamente que seus benefícios relacionados ao custo indireto, tais como melhora na qualidade de vida e produtividade das pacientes, são superiores quando comparados à realização de histerectomia (Blumenthal et al., 2011; Hurskainen et al., 2004; Spencer et al., 2017). Além disso, Da Silva Filho et al. (2016) demonstraram que pacientes brasileiras acompanhadas no contexto da saúde suplementar e tratadas com SIU-LNG 52 mg apresentam menor custo indireto, quando comparadas àquelas que realizaram histerectomia (Da Silva Filho et al., 2016). Considerando que não foram incluídos custos indiretos no modelo econômico ora apresentado, os benefícios da utilização de SIU-LNG 52 mg com diagnóstico do SUA podem ser ainda maiores. No entanto, essa hipótese precisa ser mais bem avaliada em estudos que contemplem custos indiretos.

Além dos benefícios econômicos apresentados neste estudo, a utilização de SIU-LNG 52 mg para manejo do SUA apresenta outras vantagens em relação à histerectomia, comparador considerado nesta análise. O principal aspecto está relacionado à manutenção da fertilidade, além de evitar a realização de um procedimento invasivo associado a complicações como infecção, tromboembolismo venoso e sangramento (Cheong et al., 2017; Davies \& Kadir, 2017). Van Dongen et al. (2009) conduziram um estudo com o objetivo de estimar a influência da existência de terapias alternativas à histerectomia para tratamento do SUA na frequência de realização desse procedimento cirúrgico. Após um período de seguimento de 10 anos, os autores observaram que a proporção de pacientes submetidas à histerectomia como primeira escolha de tratamento para SUA reduziu de maneira significativa de 40,6\% para 31,4\% ( $p=0,005$ ) (van Dongen et al., 2009). Dessa forma, a importância da disponibilidade de terapias alternativas à histerectomia pode ser evidenciada.

Apesar dos importantes achados apresentados nesta análise, algumas limitações devem ser destacadas. As probabilidades de transição entre os estados de saúde dos modelos foram obtidas na literatura em estudos clínicos randomizados, e tais resultados podem não representar a realidade da prática clínica diária. Além disso, os dados clínicos não são específicos para a população brasileira, havendo diferenças sociodemográficas entre as populações estudadas. 
A elaboração de microcusteio para determinação dos custos de cada componente dos modelos também pode ser considerada uma limitação, porém se torna a única opção disponível na ausência de dados publicados ou disponíveis em bases secundárias.

\section{Conclusão}

A partir das análises apresentadas neste estudo, conclui-se que o SIU-LNG 52 mg é uma alternativa dominante em relação à histerectomia para tratamento do SUA sob a perspectiva do Sistema de Saúde Suplementar no Brasil. Adicionalmente, considerando a variabilidade de custos que dificulta a extrapolação desses resultados, foi possível determinar limiares de custo do SIU-LNG 52 mg e procedimento de inserção para que possa ser observada equivalência econômica no custo de tratamento total do SUA em relação à histerectomia, de acordo com diferentes custos de cirurgia.

É possível afirmar, então, que a utilização de SIU-LNG $52 \mathrm{mg}$ para o manejo do SUA, respeitando-se os limiares de custo estabelecidos, é uma opção capaz de atrelar economia para o Sistema de Saúde Suplementar brasileiro a benefícios para a mulher em relação à cirurgia, evitando em muitos casos a necessidade de um procedimento invasivo e permitindo a manutenção da fertilidade da mulher.

\section{Agradecimentos}

Agradecemos à Origin Health pelo apoio com o desenvolvimento deste trabalho. Esse apoio foi financiado pela Bayer S.A.

\section{Referências}

Agência Nacional de Saúde Suplementar (ANS). Rol de procedimentos e eventos em saúde - Lista completa de incorporações. 2018. p. 1-2.

Associação Médica Brasileira (AMB). Classificação Brasileira Hierarquizada de Procedimentos Médicos (CBHPM). São Paulo: AMB; 2018.

Bahamondes MV, De Lima Y, Teich V, Bahamondes L, Monteiro I. Resources and procedures in the treatment of heavy menstrual bleeding with the levonorgestrel-releasing intrauterine system (LNG-IUS) or hysterectomy in Brazil. Contraception. 2012;86(3):244-50.

Barcelos RS, Zanini RV, dos Santos IS. Distúrbios menstruais entre mulheres de 15-54 anos de idade em Pelotas, Rio Grande do Sul, Brasil: Estudo de base populacional. Cad Saúde Pública. 2013;29(11):2333-46.

Barnett C, Moehner S, Do Minh T, Heinemann K. Perforation risk and intrauterine devices: results of the EURAS-IUD 5-year extension study. Eur J Contracept Reprod Heal Care. 2017;22(6):424-8.

Benetti-Pinto CL, Sá Rosa-E-Silva ACJ, Yela DA, Soares Júnior JM. Sangramento uterino anormal. Rev Bras Ginecol e Obstet. 2017;39(7):358-68.

Blumenthal PD, Dawson L, Hurskainen R. Cost-effectiveness and quality of life associated with heavy menstrual bleeding among women using the levonorgestrel-releasing intrauterine system. Int J Gynecol Obstet. 2011;112(3):171-8.

Chen YJ, Li YT, Huang BS, Yen MS, Sheu BC, Chow SN, et al. Medical treatment for heavy menstrual bleeding. Taiwan J Obstet Gynecol. 2015;54(5): 483-8.
Cheong Y, Cameron IT, Critchley HOD. Abnormal uterine bleeding. Br Med Bull. 2017;123(1):103-14.

Clegg JP, Guest JF, Hurskainen R. Cost-utility of levonorgestrel intrauterine system compared with hysterectomy and second generation endometrial ablative techniques in managing patients with menorrhagia in the UK. Curr Med Res Opin. 2007;23(7):1637-48.

Culhane J, Sharma M, Wilson K, Roberts DA, Mugo C, Wamalwa D, et al. Modeling the health impact and cost threshold of long-acting ART for adolescents and young adults in Kenya. EClinicalMedicine. 2020;25:100453.

Da Silva Filho AL, Rocha ALL, De Assis Nunes Pereira F, Tavares RL, França Ferreira MC, Lamaita RM, et al. Tratamento do sangramento uterino anormal: uma análise sob a perspectiva dos custos no Sistema Único de Saúde e da medicina suplementar. Reprod e Clim. 2016;31(1):31-6.

Davies J, Kadir RA. Heavy menstrual bleeding: An update on management. Thromb Res. 2017;151:S70-7.

Escobar-Paredes D, Peralta-Rivera R. Analysis of the cost-effectiveness of the levonorgestrel intrauterine system as alternative to histerectomies in two latin american countries. Acta Med Costarric. 2019;61(3):94-8.

Fraser IS, Mansour D, Breymann C, Hoffman C, Mezzacasa A, Petraglia F. Prevalence of heavy menstrual bleeding and experiences of affected women in a European patient survey. Int J Gynecol Obstet. 2015;128(3):196-200.

Fundação Instituto de Pesquisas Econômicas (Fipe). IPC - Índice de Preços ao Consumidor [Internet]. 2020. Available from: https://www.fipe.org.br/ pt-br/indices/ipc/\#indice-mensal\&macum

Hasselrot K, Lindeberg M, Konings P, Kallner HK. Investigating the loss of work productivity due to symptomatic leiomyoma. PLoS One. 2018;13(6):1-9.

Heinemann K, Barnett C, Reed S, Möhner S, Do Minh T. IUD use among parous women and risk of uterine perforation: a secondary analysis. Contraception. 2017;95(6):605-7.

Heinemann K, Reed S, Moehner S, Do Minh T. Risk of uterine perforation with levonorgestrel-releasing and copper intrauterine devices in the European Active Surveillance Study on Intrauterine Devices. Contraception. 2015;91(4):274-9.

Heliövaara-Peippo S, Hurskainen R, Teperi J, Aalto AM, Grénman S, Halmesmäki K, et al. Quality of life and costs of levonorgestrel-releasing intrauterine system or hysterectomy in the treatment of menorrhagia: A 10-year randomized controlled trial. Am J Obstet Gynecol. 2013;209(6):535.e1-e14.

Hurskainen R, Teperi J, Rissanen P, Aalto A, Grenman S, Kivelä A, et al. Clinical outcomes and costs with the levonorgestrel-releasing intrauterine system or hysterectomy for treatment of menorrhagia: randomized trial 5-year follow-up. JAMA. 2004;291(12):1456-63.

Instituto Brasileiro de Geografia e Estastítica (IBGE). Tábuas Completas de Mortalidade [Internet]. 2018. Available from: https://www.ibge.gov.br/ estatisticas/sociais/populacao/9126-tabuas-completas-de-mortalidade. html?=\&t=0-que-e

Jensen JT, Lefebvre P, Laliberté F, Sarda SP, Law A, Pocoski J, et al. Cost burden and treatment patterns associated with management of heavy menstrual bleeding. J Womens Health. 2012;21(5):539-47.

Louie M, Spencer J, Wheeler S, Ellis V, Toubia T, Schiff LD, et al. Comparison of the levonorgestrel-releasing intrauterine system, hysterectomy, and endometrial ablation for heavy menstrual bleeding in a decision analysis model. Int J Gynecol Obstet. 2017;139(2):121-9.

Maulsby CH, Holtgrave DR, Hamilton AB, Campbell D, Liu H, Wyatt GE. A Cost and Cost-Threshold Analysis of Implementation of an EvidenceBased Intervention for HIV-Serodiscordant Couples. AIDS Behav. 2019;23(9):2486-9. 
Ministério da Fazenda (Brasil). Banco Central do Brasil. Cotações e boletins [Internet]. 2020. Available from: https://www.bcb.gov.br/ estabilidadefinanceira/historicocotacoes

Ministério da Saúde (Brasil). Agência Nacional de Vigilância Sanitária (Anvisa). Câmara de Regulação do Mercado de Medicamentos (CMED). Preços Máximos de Medicamentos por Princípio Ativo [Internet]. 2020. Available from: https://www.gov.br/anvisa/pt-br/assuntos/ medicamentos/cmed/precos/capa-listas-de-precos

Ministério da Saúde (Brasil). Secretaria de Ciência-Tecnologia e Insumos Estratégicos. Departamento de Ciência e Tecnologia. Diretrizes metodológicas: estudos de avaliação econômica de tecnologias em saúde. 2nd ed. Brasília: Ministério da Saúde; 2014. 132p.

National Institute of Health and Care Excellence (NICE). Heavy menstrual bleeding: assessment and management. NICE Guideline NG88. Genova: NICE; 2020. 30p.

Prota F, Alves Jr. J, Villagelin D, Silva C, Chrispim A. Analysis of the costeffectiveness of the levonorgestrel releasing intrauterine system as alternative to histerectomies in private health insurance in Brazil. In: Virtual ISPOR 2020. Orlando; 2020.

Secoli SR, Nita ME, Ono-Nita SK, Nobre M. Avaliação de tecnologia em saúde II. A análise de custo-efetividade. Arq Gastroenterol. 2010;47(4):329.
Secretaria da Administração do Estado da Bahia. PlanServ. Valores Referenciais [Internet]. 2018. Available from: https://www.planserv. ba.gov.br/prestador/valores-referenciais/

Spencer JC, Louie M, Moulder JK, Ellis V, Schiff LD, Toubia T, et al. Costeffectiveness of treatments for heavy menstrual bleeding. Am J Obstet Gynecol. 2017;217(5):574.e1-e9.

Su S, Yang X, Su Q, Zhao Y. Prevalence and knowledge of heavy menstrual bleeding among gynecology outpatients by scanning a WeChat QR Code. PLoS One. 2020;15(4):1-11.

Teich V, Bahamondes MV, de Lima Y, Bolzachini Santoni N, Bahamondes $\mathrm{L}$, Monteiro I. Uso de recursos e custos associados ao tratamento da menorragia idiopática com o sistema intrauterino liberador de levonorgestrel (SIU-LNG) versus histerectomia: perspectiva do Sistema Único de Saúde (SUS). J Bras Econ Saúde. 2012:4(2):373-81.

van Dongen $H$, van de Merwe AG, de Kroon CD, Jansen FW. The impact of alternative treatment for abnormal uterine bleeding on hysterectomy rates in a tertiary referral center. J Minim Invasive Gynecol. 2009;16(1):47-51

Walker MH, Coffey W, Borger J. Menorrhagia. StatPearls. 2020.

Yela D, Benetti-Pinto C. Sangramento uterino anormal. Vol. 53, Protocolos Febrasgo. Rio de Janeiro: Febrasgo; 2018. p. 4-20. 